J. Lake Sci. (湖泊科学), 2020, 32(5): 1421-1431

DOI 10. 18307/2020. 0504

(c) 2020 by Journal of Lake Sciences

\title{
太湖蓝藻水华的年度情势预测方法探讨”
}

朱广伟 ${ }^{1 * *}$, 施 坤 ${ }^{1}$, 李 未 $^{1}$, 李 娜 ${ }^{2}$, 邹 伟 $^{1}$, 国超旋 ${ }^{1}$, 朱梦圆 ${ }^{1}$, 许 海 $^{1}$, 张运林 ${ }^{1}$, 秦伯强 ${ }^{1}$ ( 1 : 中国科学院南京地理与湖泊研究所, 湖泊与环境国家重点实验室,太湖湖泊生态系统研究站, 南京 210008)

(2: 南京师范大学虚拟地理环境教育部重点实验室,南京 210023)

\begin{abstract}
摘 要: 在太湖、巢湖、滇池、洱海、三峡水库等我国重要湖泊和水库,蓝藻水华时常发生但年际之间藻情往往有较大差 异, 给蓝藻水华的防控物资及人员投人、湖库水源地水质安全保障带来较大的挑战, 亟待探索周年尺度的蓝藻水华强度 预测方法. 本文收集了太湖连续 15 年的蓝藻水华情势观测数据和同步的气象、水文数据用于构建蓝藻水华预测模型, 提 出了利用遥感反演的蓝藻水华面积 $\left(\mathrm{A}_{\mathrm{BL}}\right)$ 及人工观测的水体浮游植物叶绿素 $a$ 浓度 $\left([\mathrm{Chl} . a]_{\mathrm{LB}}\right)$ 共同表征的蓝藻水华强 度指标 $(B I)$. 分析了太湖年尺度的 $B I$ 值与环境条件的关系, 提出了基于年初能够掌握的气象、水文、营养盐等综合环境 指标进行年度 $B I$ 预测的统计模型. 结果表明, 太湖年度 $B I$ 值与冬季及初春 (12-3 月) 日均水温 $\left(\mathrm{WT}_{12-3}\right)$ 、冬春季有效积 温 $\left(\mathrm{AT}_{12-3}\right)$ 、前一年降雨总量 $\left(\mathrm{RF}_{\mathrm{YB}}\right)$ 等环境因子呈显著正相关, 与冬季及初春的水体总氮 $\left(\mathrm{TN}_{12-3}\right)$ 、溶解性总氮 $\left(\mathrm{DTN}_{12-3}\right)$ 、总磷 $\left(\mathrm{TP}_{12-3}\right)$ 及溶解性总磷 $\left(\mathrm{DTP}_{12-3}\right)$ 不存在统计上的显著相关关系. 此外, 本研究开展了基于上述因子 $(B I$ 为因变量, 其余环境因子为自变量) 的多元 (或一元) 回归分析, 并粼选出最优模型. 总体而言, 最优模型的模拟计算结果 与实测浓度具有较高的一致性, 因此本研究得出的模型对太湖蓝藻水华年际强度预测具有较高精度. 本研究对太湖等富 营养化湖库蓝藻水华的中长期预测具有指导意义.
\end{abstract}

关键词: 蓝藻水华;季度预测;水温;降雨量;营养盐;太湖

\section{Seasonal forecast method of cyanobacterial bloom intensity in eutrophic Lake Taihu, Chi- na *}

ZHU Guangwei ${ }^{1 * *}$, SHI Kun ${ }^{1}$, LI Wei ${ }^{1}$, LI Na ${ }^{2}$, ZOU Wei $^{1}$, GUO Chaoxuan ${ }^{1}$, ZHU Mengyuan ${ }^{1}$, XU Hai ${ }^{1}$, ZHANG Yunlin ${ }^{1} \&$ QIN Boqiang ${ }^{1}$

(1: Taihu Laboratory for Lake Ecosystem Research, State Key Laboratory of Lake Science and Environment, Nanjing Institute of Geography and Limnology, Chinese Academy of Sciences, Nanjing 210008, P.R.China)

(2: Key Laboratory of Virtual Geographic Environment of Education Ministry, Nanjing Normal University, Nanjing 210023, P.R.China)

Abstract: Many important lakes and reservoirs of China, including Lake Taihu, Lake Chaohu, Lake Dianchi, Lake Erhai and Three Gorges Reservoir, were plagued with cyanobacterial blooms. However, the intensity of the blooms in these freshwaters varied significantly in different years, which exhibited significant challenges to the blooms collection organizations and drinking water plants, leading to the urgent need to cyanobacteria blooms prediction model based on annual dataset. Therefore, the long-term ( 15 years ) observation data and meteorological and hydrological datasets of Lake Taihu were collected for the prediction of algal blooms. In current study, cyanobacterial bloom intensity index $(B I)$ were proposed with the consideration of yearly average blooms area interpret by high frequency remote sensing images and whole lake average chlorophyll- $a$ concentration. Furthermore, environmental factors, such as water temperature, rainfall, water level, nitrogen and phosphorus concentrations were used as the crucial factors to predict BI. Our results showed that average water temperature in winter and early spring, as well as the rainfall of the former year were significant positive factors of the yearly $B I$ value in Lake Taihu. While the nutrient-related factors in early spring had no signif-

* 2020-02-10 收稿; 2020-03-16 收修改稿.

国家自然科学基金项目 (41671494,41830757)、国家水体污染控制与治理科技重大专项(2017ZX07203001)、中国科学 院前沿科学重点研究项目 (QYZDJ-SSW-DQC008) 和山东省重大科技创新工程项目 (2018YFJH0902) 联合资助.

** 通信作者;E-mail: gwzhu@ niglas.ac.cn. 
icant relationships with $B I$. In addition, a multiple (or univariate) regression analysis based on the above factors ( $B I$ was the dependent variable and the remaining environmental factors were the independent variables) were performed in this study, and the optimal model was selected. In general, the predicted results of the selected optimal model had a high consistency with the measured concentrations, thus the model obtained in this study had relatively high accuracy for predicting the interannual intensity of cyanobacteria blooms in Taihu Lake. This study may serve reliably for the medium- and long-term prediction of cyanobacteria blooms in Lake Taihu, and other eutrophic lakes.

Keywords: Cyanobacterial blooms; seasonal forecast; water temperature; rainfall, nutrient; Lake Taihu

在持续高投入治理 10 年之后, 太湖蓝藻水华仍处于高发态势. 特别是 2017 年, 太湖的蓝藻水华强度和 水体总磷浓度双双反弹, 给太湖蓝藻水华防控及周边城市饮用水安全保障带来了挑战 ${ }^{[1]}$. 除了太湖之外, 国 内外多个类似大型湖库的蓝藻水华暴发强度都出现了高强度治理背景下的反复. 如巢湖近年来的治理投人 大,蓝藻水华控制收效不佳,2012-2018 年间,巢湖水质改善的效果变缓,虽然氨氮浓度继续下降,但 2018 年蓝藻水华的面积反而增加, 水体总氮、总磷浓度也反弹增高 ${ }^{[2]}$. 我国浙江省富春江水库, 在 “五水共治” 水 污染治理行动全面铺开、河道水体营养盐开始下降的情况下, 却于 2016 年 8 月发生了较大规模的蓝藻水华 状况 ${ }^{[3]}$. 美国伊利湖自 $1960 \mathrm{~s}$ 实施了严格的营养盐控制措施,成功控制蓝藻水华数十年,但近年来蓝藻水华 再次出现,2011 年甚至暴发了历史上最大规模的蓝澡水华 ${ }^{[4]}$; 2014 年 8 月 1 日, 因蓝藻水华引起的水厂出水 藻毒素超标, 伊利湖畔 60 万人口的城市 Toledo 中断供水 2 天 ${ }^{[5]}$. 即使在水质优良的贝加尔湖, 2016 年 7 月 26 日也发生了数平方千米的长狍藻 (Dolichospermum) 水华 ${ }^{[6]}$. Ho 等通过遥感反演的方法评价了全球 85 个 重要湖库过去 30 年的蓝澡水华变化趋势, 发现大多数水体的蓝藻水华问题仍在加重 ${ }^{[7]}$. 这些现象都表明, 即使人类控制湖库富营养化的力度在加强, 但是由于人类活动产生的污染负荷增加, 损害湖库流域自净能 力的土地扰动强度增加, 气候变暖等有利于发生藻类水华的自然环境条件在恶化, 湖泊和水库等重要水体 的蓝藻水华风险仍将在较长时间内存在. 研发湖库蓝藻水华风险预测技术, 提前对湖泊和水库的蓝藻水华 发生强度及其引发的环境风险实施预测,指导管理部门充分应对,显得十分必要.

关于藻类水华灾害的预测预警技术, 起步于 20 世纪末, 近年来得到快速发展. 尤其是短期预测预报技 术的发展迅速. 1999 年, 美国新泽西州 Rutgers 大学海洋与海岸带研究所的 Schofield 等提出了利用遥感技术 与水体光学探头原位监测为信息支撑, 采用简单推算模型的方法实施海洋赤潮短期预测的概念 ${ }^{[8]}$. 随后, 香 港大学的 Wong 等在对近海赤潮短期预报的机理模型及条件阈值等方面进行了系统完善, 并于 2000-2003 年在香港海湾实践了赤潮预测 ${ }^{[9]}$. 美国海洋与大气局 (NOAA) 依托大尺度遥感监测信息, 从 2004 年开始对 墨西哥湾的腰鞭毛藻 (Karenia brevis) 赤潮进行预测, 从 2008 年起对缅因湾芬地亚历山大藻 (Alexandrium fundyense) 赤潮实施预测, 2011 年开始对伊利湖的微囊藻 (Microcystis) 水华实施预测 ${ }^{[10]}$. Tian 等则在深圳湾 构建了完善的原位监测一遥感监测一水动力生态模型及基于 GIS 系统的结果展示海湾赤潮监测预测系统 HMFS, 实现了 $5 \mathrm{~d}$ 的大面积海域赤潮短期预报 ${ }^{[11]}$. 由于海湾环境变化和流场变化的复杂性, 关于海湾的赤 潮预测 , 目前都是一周以内的短期预报,未见有中长期预测的研究报道.

内陆水体的藻类水华预测技术方面, 孔繁翔、秦伯强、李未等在太湖开展了大量的探索. 通过对太湖蓝 藻水华不同生长阶段限制因子的辨识, 孔繁翔等提出了以卫星遥感数据和湖面人工巡测为数据驱动、以未 来 1 3 d 气象条件为预测情景条件的太湖蓝藻水华短期预测预警方法, 并从 2007 年 7 月起在太湖开始应 用 ${ }^{[12]}$. 秦伯强、李未等则从蓝藻水华漂移堆积的水动力过程精准模拟人手, 开发了基于三维水动力模型支 撑的太湖蓝藻水华预测预警系统并长期实施业务化运行 ${ }^{[13-15]}$. 叶麟和蔡庆华则基于逐日单点水质及环境参 数的监测, 开发了利用人工神经网络 (ANN) 预测未来 $7 \mathrm{~d}$ 的大型水库支汉蓝藻水华风险预测方法 ${ }^{[16]}$. 此后, 大量关于湖库蓝藻水华短期预测的方法被报道. 这其中绝大多是基于模型算法方面的短期预测尝试和 改进 ${ }^{[17-21]}$.

关于年际尺度、季度尺度的中长期蓝藻水华情势预报技术的报道很少. 与海湾相比, 湖泊和水库生态系 统更加封闭, 环境条件更加稳定, 具备在更长尺度上实施蓝藻水华强度预测的可行性. 孔繁翔等根据蓝藻水 华形成的“四阶段假说”理论, 基于 2007 年的蓝藻种源情况、影响蓝藻水华强度的水环境现状及 2008 年的 水文和气候预测, 对 2008 年的蓝藻水华开始时间、夏季强度及秋季结束时间等进行了定性预测 ${ }^{[12]}$. 美国海 
洋与大气局的 Stumpf 等从 2012 年开始对伊利湖的蓝藻水华情势实施了提前一个月到提前一个季度的预测 尝试 ${ }^{[22-23]}$. 通过构建春季经由西伊利湖主要污染人流河道 Maumee 河的人湖有效磷负荷与夏季蓝藻水华强 度之间的关系, 建立了伊利湖夏季蓝藻水华强度的定量预测方法 ${ }^{[23]}$. Obenour 等在其基础上, 通过贝叶斯层 次模型进一步优化了预测模型的应用范围,将水华期藻类叶绿素 $a$ 浓度与春季磷负荷的响应关系细分为 3 类, 分类使用不同模型进行预测, 提升了模型预测的精度 ${ }^{[24]}$. 尽管该预测结果不尽理想, 但是该预测的思路 为进一步开发中长期蓝藻水华预测技术提供了借鉴.

太湖是国际上关于湖泊蓝藻水华情势及环境条件观测记录最完整的湖泊之一. 基于太湖湖泊生态系统 研究站 (简称 “太湖站”) 的观测记录数据库, 回溯太湖蓝藻水华年度强度与环境因子之间的关系, 有望建立 切实可行的湖泊蓝藻水华季度/年度情势预测方法. 因此, 本文通过对 2005-2019 年太湖蓝藻水华情势及 相关水质、水文、气象关系的统计分析, 试图构建利用早春气象、水文及营养盐条件与年度蓝藻水华强度之 间的定量关系, 探索一种能对全年蓝藻水华情势实施定量预判的方法, 为太湖及类似的富营养湖库藻类水 华防控提供理论支撑.

\section{1 材料与方法}

\section{1 年度蓝藻水华强度估算}

采用两种信息综合对太湖的年度蓝藻水华强度进行评估. 第一, 太湖年度水体浮游植物叶绿素 $a$ 人工 监测的加权平均浓度. 这是一种能够定量反映全湖全年浮游植物生物量的指标. 本文采用太湖站每年 2 月、 5 月、8 月、11 月 4 次全太湖 32 个观测点采样( 分上、中、下 3 层采样后混合水样) 分析获得的水体浮游植物 叶绿素 $a$ 浓度, 按照泰森多边形的方式获得各个点位的水量权重, 加权计算出全年水体浮游植物叶绿素 $a$ 浓度平均值, 计作 $[\text { Chl. } a]_{\mathrm{LB}}$, 具体布点及加权平均值计算方法见文献 $[25]$. 第二, 采用每月多幅遥感影像获 得的全年太湖蓝藻水华面积平均值, 记作 $\mathrm{A}_{\mathrm{BL}}$. 这是一种定量精准反映全湖水华分布及其动态变化的指标. 本文采用的太湖蓝藻水华面积及其叶绿素 $a$ 的遥感反演方法见文献 [26], 遥感影像数据采用 MODIS aqua L-0 数据, 2005 年 1 月 1 日- 2019 年 12 月 31 日的 5000 余景的逐日影像从 NASA 的 Goddard Space Flight Center Web 网站下载 (oceancolor.gsfc.nasa.gov), 经目视隧选, 共获得太湖湖面无云可用的 1522 景影像实施 水体叶绿素 $a$ 浓度反演, 反演的叶绿素 $a$ 浓度记作 $[\text { Chl. } a]_{\mathrm{RS}}$, 其反演算法 ${ }^{[27]}$ 为:

$$
[\text { Chl. } a]_{\mathrm{RS}}=-1545.3-[(\operatorname{EXP}(\operatorname{Rrc}(645))-\operatorname{EXP}(\operatorname{Rrc}(859))) /
$$

$$
(\operatorname{EXP}(\operatorname{Rrc}(645))+\operatorname{EXP}-(\operatorname{Rrc}(859)))]+69.346
$$

在本文中, $[\mathrm{Chl} . a]_{\mathrm{LB}} 、[\mathrm{Chl} . a]_{\mathrm{RS}} 、 \mathrm{~A}_{\mathrm{BL}}$ 均可以在一定程度上反映当年的蓝藻水华强度. 为了更为客观地反 映蓝藻水华的藻类生物量和水华面积特征, 构建蓝藻水华强度指数 $B I$ (Bloom Intensity), 定义 $B I$ 为 $[\text { Chl. } a]_{\mathrm{LB}}$ 与 $A_{\mathrm{BL}}$ 的乘积与统计期间 (2005-2019 年) 该值最大值的比值.

$$
B I=\frac{\left(\mathrm{A}_{\mathrm{BL}} \cdot[\mathrm{Chl} . a]_{\mathrm{LB}}\right)_{i}}{\left(\mathrm{~A}_{\mathrm{BL}} \cdot[\mathrm{Chl} . a]_{\mathrm{LB}}\right)_{\max }} \times 100
$$

式中, $i$ 是具体某年份, 而 $\max$ 则是 2005-2019 年的最大值, 为 2017 年. 按此公式, 2017 年的 $B I$ 值为 100 , 其 余年份与之相比. 对未来值而言, $B I$ 值允许大于 100 .

\section{2 相关环境因子数据及其来源}

根据施坤等对 2003-2017 年太湖蓝藻水华物候学的分析 ${ }^{[26]}$, 温度、降雨、风速、营养盐等均为影响太湖 蓝藻水华情势变化的重要环境条件. 从预测的操作性方面, 我们分别从气象、水文、营养盐 3 个方面考虑影 响年度太湖蓝藻水华强度的环境因子.

气象要素方面, 从太湖湖泊生态系统观测站获得 2004 年 1 月 1 日一 2019 年 12 月 31 日太湖站气象观测 场逐日降雨量数据. 由于前期的降雨量大小直接影响了外源引发的湖泊营养盐的积蓄程度, 我们分 3 个时 段进行分析, 前一年降雨总量 $\left(\mathrm{RF}_{\mathrm{YB}}, \mathrm{mm}\right)$, 前一年 12 月至当年 3 月降雨总量 $\left(\mathrm{RF}_{12-3}, \mathrm{~mm}\right)$, 以及前一年 9 月 至当年 2 月降雨总量 $\left(\mathrm{RF}_{9-2}, \mathrm{~mm}\right)$. 之所以强调 12-3 月的累积量, 是因为传统上该时段是藻类生长的缓慢 期, 也是蓝藻快速增长的物质和能量积蓄期.

水文数据方面,采用了太湖站 2004 年 1 月 1 日- 2019 年 12 月 31 日逐日观测的水位 (WL) 数据及水下 
$50 \mathrm{~cm}$ 的水温 (WT) 数据. 其中水位方面, 提取了前一年 12 月至当年 3 月的逐日水位平均值 $\left(\mathrm{WL}_{12-3}, \mathrm{~m}\right)$. 水 温方面, 也提取了前一年 12 月至当年 3 月的逐日水温平均值 $\left(\mathrm{WT}_{12-3},{ }^{\circ} \mathrm{C}\right)$. 此外, 根据孔繁翔等观测提出的 野外 $9^{\circ} \mathrm{C}$ 以上太湖蓝藻开始复苏的报道 ${ }^{[12]}$, 也尝试了积温统计: 也即, 将 $12-3$ 月期间, 每天的水温减去 $9^{\circ} \mathrm{C}$, 然后, 将其中的正值部分进行累加, 获得该时段的水温有效积温, 记作 $\mathrm{AT}_{12-3}$; 同理, 依据 1 月之后水温 开始大于 $9^{\circ} \mathrm{C}$ 起至 3 月底的逐日水温获得的有效积温记作 $\mathrm{AT}_{\mathrm{s}}$, 依据 2 月和 3 月的逐日水温获得的有效积温 记作 $\mathrm{AT}_{2-3}$.

水质数据方面, 根据前期的研究报道, 氮和磷均能影响太湖蓝藻生物量的变化 ${ }^{[28]}$, 因此, 综合考虑了水 体氮、磷浓度. 但由于水体总氮 ( TN) 、总磷 (TP) 浓度很大程度上受水体藻类生物量反馈的影响 ${ }^{[29]}$, 因此, 还 特别采集了北太湖具有代表性监测点位的溶解性总氮 (DTN) 和溶解性总磷 (DTP) 浓度表征水体营养盐因 素, 在太湖站逐月监测的北太湖 14 个监测点中, 剔除 THL00 和 THL06 两个分别位于梅梁湾梁溪河口和直湖 港河口的点位, 将其余 12 个点位的 TN、DTN、TP、DTP 进行月值数学平均, 然后, 再将前一年 12 月至当年 3 月共计 4 个月的数学平均值求出, 分别记作 $\mathrm{TN}_{12-3} 、 \mathrm{DTN}_{12-3} 、 \mathrm{TP}_{12-3}$ 和 $\mathrm{DTP}_{12-3}$.

\section{3 数据统计处理}

蓝藻水华强度与相关环境因子之间的定量关系分析, 采用 SPSS 多元回归分析中的进人回归法依次分 析. 各环境因子与蓝藻水华强度的关系, 采用相关分析进行比选.

\section{2 结果}

\section{$2.12005-2019$ 年太湖蓝藻水华强度变化}

$2005-2019$ 年太湖水体 $[\text { Chl. } a]_{\mathrm{LB}} 、 A_{\mathrm{BL}} 、[\text { Chl. } a]_{\mathrm{RS}}$ 以及 $B I$ 的变化如图 1. 从图中可以看出, 2017 年所有 指标出现峰值, 年均蓝藻水华面积达到 $284.28 \mathrm{~km}^{2}$, 是 15 年平均值 $\left(180.62 \mathrm{~km}^{2}\right)$ 的 1.6 倍; 年均 $[\mathrm{Chl} . a]_{\mathrm{LB}}$ 达 到 $40.8 \mu \mathrm{g} / \mathrm{L}$, 是 15 年平均值 $(19.6 \mu \mathrm{g} / \mathrm{L})$ 的 2.1 倍.

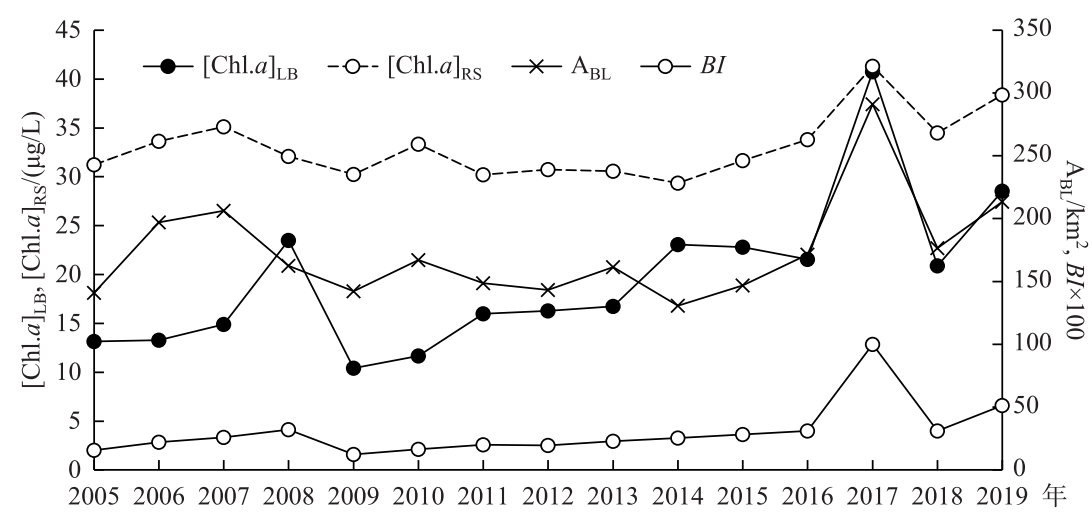

图 $12005-2019$ 年太湖蓝藻水华强度指标变化

Fig.1 Variation of annual cyanobacterial bloom intensity in Lake Taihu from 2005 to 2019

表征蓝藻水华年度强度的指标 $[\text { Chl. } a]_{\mathrm{LB}}$ 和 $\mathrm{A}_{\mathrm{BL}}$ 在年度变化上并不完全一致. 水华面积 $\mathrm{A}_{\mathrm{BL}}$ 在 2007 年和 2006 年分别出现第 2 峰值和第 3 峰值, 而反映藻类生物量的指标 $[\mathrm{Chl} . a]_{\mathrm{LB}}$ 则分别在 2008 年、2014 年等形成 与面积变化不同步的峰值. 2008 年和 2014 年浮游植物叶绿素 $a$ 浓度的反常增高, 与同期藻类群落结构变 化,特别是硅藻门生物量的明显增高有关 ${ }^{[30]}$. [Chl. $\left.a\right]_{\mathrm{LB}}$ 与 $[\mathrm{Chl} . a]_{\mathrm{RS}}$ 在变化趋势上基本一致,但遥感反演的 值明显偏高, $[\mathrm{Chl} . a]_{\mathrm{LB}}$ 与 $[\mathrm{Chl} . a]_{\mathrm{RS}}$ 多年均值分别为 19.6 和 $33.1 \mu \mathrm{g} / \mathrm{L}$. 遥感值偏高是由于蓝藻水华物质的 表层聚集现象所致, 表层水体藻类生物量高于水柱平均值是合理的. 此外, 二者的相关性非常好, 相关系数 达到 0.701 $(P<0.004, n=15)$.

\section{2 相关环境因子变化}

2005-2019 年与蓝藻水华情势密切关联的气象、水文、营养盐等相关指标见表 1 . 从表中可以看出, 与 
$B I$ 显著相关的指标包括 $\mathrm{RF}_{\mathrm{YB}} 、 \mathrm{RF}_{9-2} 、 \mathrm{WT}_{12-3} 、 \mathrm{AT}_{12-3}$ 等. 这反映出水温、降雨等环境变化对年度太湖蓝藻水华 强度具有较强的决定作用. 而冬、春季节的水体 TN、DTN、TP、DTP 等指标甚至与 $B I$ 呈不显著的弱的负相关 关系. 2005-2019 年太湖冬季及早春 (12-3 月) 逐日水温、水位、降雨量以及前一年的降雨总量变化如图 2 所示. 可以看出,就 $12-3$ 月的平均水温而言, 15 年来的平均值为 $8.50^{\circ} \mathrm{C}$,其中 2007 年、2008 年、2017 年和 2019 年的平均水温超过 $9^{\circ} \mathrm{C}$, 也即微囊藻能够开始生长的野外临界温度 ${ }^{[12]}$. 最大值出现在 2017 年, 为 $9.83^{\circ} \mathrm{C}$, 比平均值高 $16 \%$. 第 2 高值出现在 2007 年, 为 $9.73^{\circ} \mathrm{C}$, 第 3 高值出现在 2019 年, 为 $9.45^{\circ} \mathrm{C}$,第 4 高值 出现在 2008 年, 为 $9.38^{\circ} \mathrm{C}$. 最低值出现在 2012 年, 为 $7.40^{\circ} \mathrm{C}$. 与图 1 比较能够看出, 2007 年和 2017 年的太 湖蓝藻水华大暴发, 都经历了冬季及早春的水温异常偏高, 这与之前的分析结果一致 ${ }^{[29]}$. 同时表明,在进行 太湖这种营养盐本底较高的湖泊水华情势预测时,水温是最应关注的环境要素.

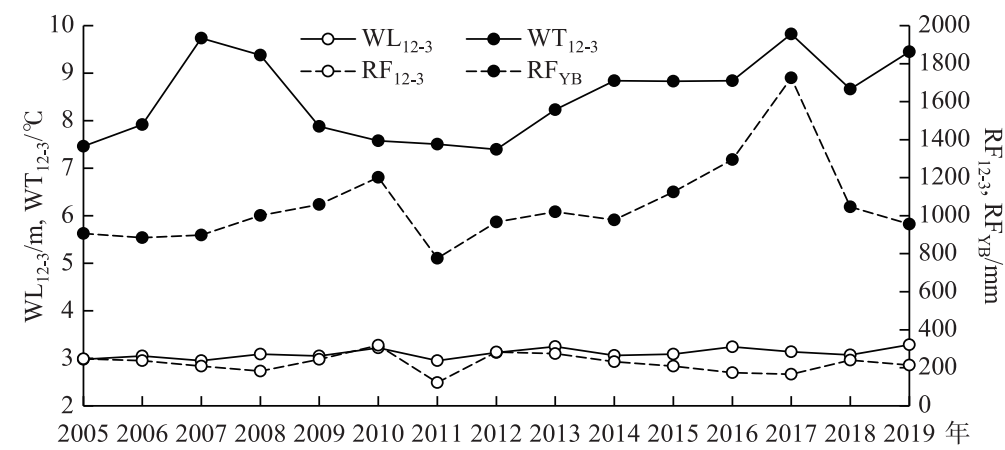

图 2 2005-2019 年每年 12-3 月太湖日均水温、日均水位、累积降雨量及前一年降雨总量的变化

Fig.2 Variation of daily average water temperature, water level, accumulate rainfall during December and March, and rainfall of the ahead year in Lake Taihu from 2005 to 2019

与水温均值类似的参数积温, 表现出与水温变化类似的趋势, 也均与 $[\mathrm{Chl} . a]_{\mathrm{L} \mathrm{B}}$ 等年度水华强度数据呈正 相关 (图 3). 相比较而言, 12-3 月的逐日水温均值 $\mathrm{WT}_{12-3}$ 与全年全湖平均浮游植物叶绿素 $a$ 浓度 $[\text { Chl. } a]_{\mathrm{LB}}$ 的 相关系数明显强于积温. $\mathrm{AT}_{12-3}$ 尽管与 $[\mathrm{Chl} . a]_{\mathrm{LB}}$ 仍呈显著相关,但是相关系数明显小于 $\mathrm{WT}_{12-3.3}$. 这表明,整个冬 季的水温状况,而非仅仅是高于微囊藻复苏生长部分的积温,对全年藻类生物的影响更为重要.

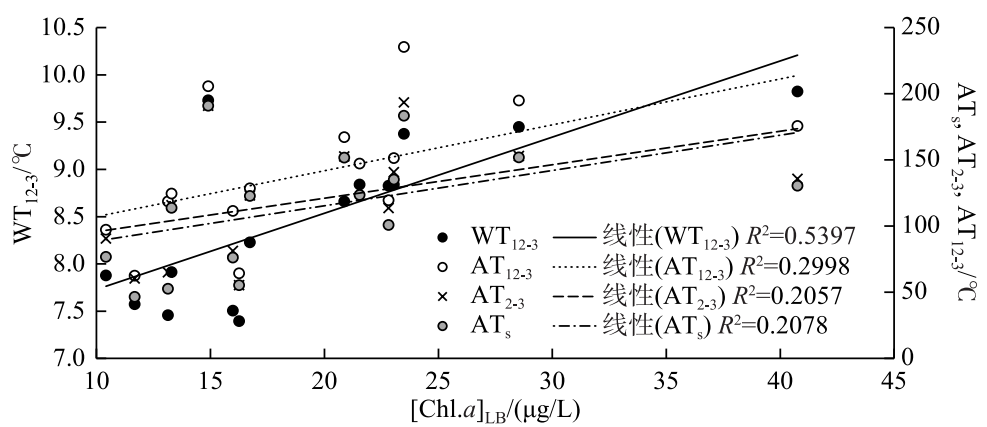

图 3 冬、春季水温相关指标与 $[\text { Chl. } a]_{\mathrm{LB}}$ 的相关性

Fig.3 Relationship between [Chl. $a]_{\text {LB }}$ and water temperature parameters in winter and early spring

与之前的报道类似, 北太湖水体 TN、DTN 浓度在 2006 年峰值以后, 呈现不断下降的趋势 ${ }^{[29]}$. 冬、春季是 太湖水体 TN 浓度较高的季节, 12-3 月的北太湖均值在 2006 年为 $5.88 \mathrm{mg} / \mathrm{L}, 2019$ 年下降为 $2.95 \mathrm{mg} / \mathrm{L}$, 首 次降至 $3 \mathrm{mg} / \mathrm{L}$ 以下, 下降幅度接近 50\%. DTN 浓度的下降幅度更大, 达到 59\% (表 1). 然而, 冬、春季水体 $\mathrm{TN}$ 浓度的下降与蓝藻水华强度变化和水体 $\mathrm{TP}$ 浓度变化很不一致, 说明在当前的 $\mathrm{TN}$ 浓度范围内, 氮的变化 不是影响太湖藻类生物量的关键因素. 
冬、春季( $12-3$ 月) 北太湖 TP 浓度的最高值出现在 2009 年, 为 $0.151 \mathrm{mg} / \mathrm{L}$. 此后基本呈下降趋势, 2017 年为 $0.113 \mathrm{mg} / \mathrm{L}$, 与其他年份相比, 水华异常严重的 2017 年年初水体 TP 浓度并不特别高. 这也从一个 方面表明冬、春季水体磷浓度对夏季蓝藻水华情势影响也有限 (表 1). 2019 年冬、春季北太湖 TP 浓度为 $0.102 \mathrm{mg} / \mathrm{L}$, 接近近 15 年来的最低值,与当年较为严重的蓝藻水华现象也不一致 (表 1 ).

表 $12005-2019$ 年太湖蓝藻水华情势相关环境因子及其与年度水华强度 $B I$ 的相关性分析 ${ }^{1)}$

Tab.1 Environmental factors related to cyanobacterial bloom intensity in Lake Taihu from 2005 to 2019

\begin{tabular}{ccccccccccccc}
\hline 年份 & $\mathrm{RF}_{12-3}$ & $\mathrm{RF}_{\mathrm{YB}}$ & $\mathrm{RF}_{9-2}$ & $\mathrm{WT}_{12-3}$ & $\mathrm{AT}_{12-3}$ & $\mathrm{AT}_{2-3}$ & $\mathrm{AT}_{\mathrm{s}}$ & $\mathrm{WL}_{12-3}$ & $\mathrm{DTN}_{12-3}$ & $\mathrm{TN}_{12-3}$ & $\mathrm{DTP}_{12-3}$ & $\mathrm{TP}_{12-3}$ \\
\hline 2005 & 246.24 & 906.35 & 372.98 & 7.46 & 118.81 & 65.11 & 52.82 & 2.977 & 4.58 & 5.17 & 0.031 & 0.105 \\
2006 & 237.80 & 885.00 & 478.40 & 7.91 & 124.76 & 115.92 & 113.88 & 3.047 & 5.14 & 5.88 & 0.049 & 0.140 \\
2007 & 209.00 & 898.33 & 299.80 & 9.73 & 205.85 & 191.04 & 191.04 & 2.950 & 5.04 & 5.53 & 0.056 & 0.121 \\
2008 & 181.80 & 1001.00 & 386.20 & 9.38 & 235.41 & 193.51 & 183.47 & 3.089 & 4.40 & 4.88 & 0.078 & 0.146 \\
2009 & 244.40 & 1059.20 & 397.80 & 7.88 & 97.41 & 90.63 & 76.79 & 3.047 & 3.06 & 3.67 & 0.070 & 0.151 \\
2010 & 317.60 & 1201.40 & 333.60 & 7.58 & 62.71 & 60.56 & 46.62 & 3.217 & 4.09 & 4.46 & 0.050 & 0.112 \\
2011 & 121.80 & 776.40 & 191.80 & 7.51 & 111.51 & 81.27 & 76.40 & 2.951 & 3.25 & 3.80 & 0.063 & 0.142 \\
2012 & 283.00 & 967.00 & 202.00 & 7.40 & 64.42 & 55.50 & 55.50 & 3.121 & 3.14 & 3.61 & 0.064 & 0.134 \\
2013 & 274.80 & 1020.60 & 423.20 & 8.23 & 128.68 & 123.25 & 122.90 & 3.245 & 3.30 & 3.76 & 0.063 & 0.123 \\
2014 & 231.80 & 977.10 & 369.00 & 8.84 & 151.43 & 140.87 & 135.34 & 3.059 & 2.97 & 3.48 & 0.049 & 0.116 \\
2015 & 208.80 & 1124.80 & 259.80 & 8.83 & 119.75 & 113.67 & 100.88 & 3.084 & 2.97 & 3.44 & 0.057 & 0.128 \\
2016 & 173.40 & 1295.20 & 371.60 & 8.84 & 147.31 & 127.12 & 123.65 & 3.238 & 2.72 & 3.18 & 0.043 & 0.107 \\
2017 & 166.00 & 1725.40 & 645.20 & 9.83 & 175.79 & 135.77 & 130.65 & 3.137 & 2.52 & 3.10 & 0.038 & 0.113 \\
2018 & 239.40 & 1046.05 & 470.80 & 8.66 & 167.30 & 152.82 & 151.88 & 3.069 & 2.52 & 3.02 & 0.040 & 0.101 \\
2019 & 213.80 & 956.40 & 368.00 & 9.45 & 194.97 & 153.12 & 151.98 & 3.287 & 2.11 & 2.95 & 0.045 & 0.102 \\
$R$ & -0.425 & $0.756 *$ & $0.650 * *$ & $0.735 * *$ & $0.682 * *$ & 0.473 & 0.369 & 0.290 & -0.426 & -0.394 & -0.357 & -0.326 \\
$P$ & 0.114 & 0.001 & 0.009 & 0.002 & 0.005 & 0.075 & 0.176 & 0.295 & 0.114 & 0.146 & 0.191 & 0.236 \\
\hline
\end{tabular}

1) $R F$ 的单位为 $m m$, 特别注意 $R F_{Y B}$ 是指前一年的降雨量; $W T 、 A T$ 的单位为 ${ }^{\circ} \mathrm{C} ; \mathrm{WL}$ 的单位为 $m$; DTN、TN、DTP、TP 的单位 为 $\mathrm{mg} / \mathrm{L} . R$ 及 $P$ 指该参数与 $B I$ 的相关系数及显著性差异参数,其中 $*$ 表示显著相关, $* *$ 表示极显著相关.

\section{3 预测模型构建}

根据表 1 和图 3 , 篮选了 $\mathrm{RF}_{\mathrm{YB}} 、 \mathrm{WT}_{12-3} 、 \mathrm{DTN}_{12-3} 、 \mathrm{WL}_{12-3} 、 \mathrm{TP}_{12-3} 5$ 个指标作为太湖蓝藻水华周年强度 $B I$ 的 预测因子, 采用依次增加的方式进行逐步回归, 比较各因子加人后对太湖年度水华强度的预测能力. 对数据 进行正态分布分析发现, 除 $\mathrm{RF}_{\mathrm{YB}}$ 之外的指标均符合正态分布. 为此, 先对所有的数据取自然对数, 然后再进 行回归分析. 各回归方程及决定系数 $R^{2}$ 见表 2.

表 2 不同环境因子组合对太湖年度水华强度 $B I$ 的预测关系式

Tab.2 Regression equation between $B I$ and environmental factors in Lake Taihu

\begin{tabular}{llr}
\hline 人选环境因子 & 回归方程 & $R^{2}$ 及显著性 \\
\hline (1) $\mathrm{WT}_{12-3}$ & $\ln (B I)=3.92 \ln \left(\mathrm{WT}_{12-3}\right)-5.11$ & $0.611,0.001$ \\
$(2) \mathrm{RF}_{\mathrm{YB}}$ & $\ln (B I)=1.54 \mathrm{Ln}\left(\mathrm{RF}_{\mathrm{YB}}\right)-7.47$ & $0.333,0.024$ \\
$(3) \mathrm{WT}_{12-3}+\mathrm{RF}_{\mathrm{YB}}$ & $\ln (B I)=3.30 \ln \left(\mathrm{WT}_{12-3}\right)+0.87 \ln \left(\mathrm{RF}_{\mathrm{YB}}\right)-9.83$ & $0.702,0.001$ \\
$(4) \mathrm{WT}_{12-3}+\mathrm{RF}_{\mathrm{YB}}+\mathrm{DTN}_{12-3}$ & $\ln (B I)=3.14 \mathrm{Ln}\left(\mathrm{WT}_{12-3}\right)+0.64 \ln \left(\mathrm{RF}_{\mathrm{YB}}\right)-0.44 \ln \left(\mathrm{DTN}_{12-3}\right)-7.38$ & $0.748,0.001$ \\
$(5) \mathrm{WT}_{12-3}+\mathrm{RF}_{\mathrm{YB}}+\mathrm{DTN}_{12-3}+$ & $\ln (B I)=3.14 \ln \left(\mathrm{WT}_{12-3}\right)+0.65 \ln \left(\mathrm{RF}_{\mathrm{YB}}\right)-0.45 \ln \left(\mathrm{DTN}_{12-3}\right)-$ & $0.748,0.005$ \\
$\mathrm{WL}_{12-3}$ & $0.03 \ln \left(\mathrm{WL}_{12-3}\right)-7.35$ & $0.752,0.014$ \\
(6) $\mathrm{WT}_{12-3}+\mathrm{RF}_{\mathrm{YB}}+\mathrm{DTN}_{12-3}+$ & $\ln (B I)=3.08 \ln \left(\mathrm{WT}_{12-3}\right)+0.64 \ln \left(\mathrm{RF}_{\mathrm{YB}}\right)-0.41 \ln \left(\mathrm{DTN}_{12-3}\right)-$ & \\
$\mathrm{WL}_{12-3}+\mathrm{TP}_{12-3}$ & $0.26 \ln \left(\mathrm{WL}_{12-3}\right)-0.28 \ln \left(\mathrm{TP}_{12-3}\right)-7.54$ & \\
\hline
\end{tabular}

对比表 2 中回归方程的决定系数, 方程 3 和 4 具有明显的优势, 特别是方程 4 , 相关系数已经接近最大 值, 整个公式的显著性较高, 人选参数简单易测. 而更多参数的方程, 如方程 5 和 6 , 对方程的精度提高有限, 
但增加了更多的参数需求, 也增加了不确定性. 为此, 采用 方程 4 较为合理. 对比方程 3 和方程 4 拟合值与实际值可 以看出,包含了水温、降雨量和 DTN 3 个环境参数的方程 4 拟合值与实测值相关性优于只有水温和降雨量 2 个参 数的方程 3(图 4).

\section{3 讨论}

\section{1 预测模型参数选择的合理性分析}

越来越多的研究表明,水文气象因素是太湖等富营养 化湖库蓝藻水华强度的主导因素. 影响湖泊浮游植物生长 的主要因子包括光、温等气象条件,水动力、换水周期等水

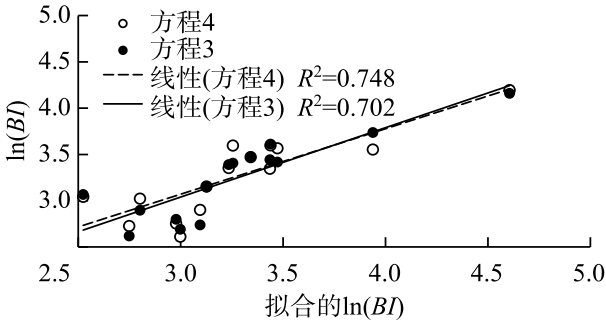

图 4 太湖年度蓝藻水华强度实测值与 方程拟合值的关系

Fig.4 Relationship between calculated $B I$ and simulated $B I$ of Lake Taihu 文条件, 氮、磷、碳、铁、硅等营养盐条件, 以及浮游动物、鱼 类、大型水草等生态系统结构状况等. 王菁晗等统计了太湖、巢湖、滇池 35 年来的气象、水质因子的区别, 发 现适合藻类水华发生的时间长是滇池蓝藻水华较太湖、巢湖严重的原因之一 ${ }^{[31]}$. 太湖的水体营养盐条件目 前还明显高于能够限制浮游植物生长、蓝藻水华暴发的临界条件. 据许海等的原位浮游植物生长的营养盐 添加实验判断, 当水体溶解性磷浓度达到 $0.03 \mathrm{mg} / \mathrm{L}$ 、溶解性氮浓度达到 $0.8 \mathrm{mg} / \mathrm{L}$ 时, 对藻类生物量增加的 影响就不明显了, 也即达到该浓度水体就能完全满足湖水中藻类快速增殖的需要 ${ }^{[28]}$. 2005-2019 年太湖全 湖水体 DTP 浓度均值为 $0.053 \mathrm{mg} / \mathrm{L}$, DTN 浓度均值为 $3.45 \mathrm{mg} / \mathrm{L}$. 其中 2019 年的年均值分别为 0.045 和 $2.110 \mathrm{mg} / \mathrm{L}$, 分别高出临界值 $50 \%$ 和 $164 \%$, 因此, 太湖全湖营养盐浓度并未低到能够对浮游植物生长产生 限制的水平.

就空间上而言, 2017 年和 2019 年太湖年均水体 DTN 和 DTP 浓度如图 5. 统计表明, 2017 年和 2019 年 DTN 浓度低于 $0.8 \mathrm{mg} / \mathrm{L}$ 的面积分别为 109 和 $317 \mathrm{~km}^{2}$, 也即藻类生长能够受到氮限制的区域增加了 208 $\mathrm{km}^{2}$, 约占太湖总面积的 $10 \%$. 而 2017 年和 2019 年 DTP 浓度低于 $0.03 \mathrm{mg} / \mathrm{L}$ 的区域面积分别为 1703 和 $1544 \mathrm{~km}^{2}, 2019$ 年受到磷限制的水域面积反而减少了 $159 \mathrm{~km}^{2}$, 凸显了太湖水体磷控制的复杂性. 从表 1 可 知, 太湖水体 DTN 浓度与全年藻类浮游植物浓度甚至呈不显著的弱的负相关, 并不表明蓝藻水华强度的增 加与氮的下降有关. 有两种可能的解释: (1) 15 年来太湖的治理带来的营养盐下降效应, 被气温增加、雨量 增加等对湖泊蓝藻水华生物量影响更大的环境因子的不利影响所抵消, 出现了水体 DTN 浓度不断下降, 但 湖泊水体浮游植物叶绿素 $a$ 浓度反而升高的结果, 这种负相关, 不代表二者具有因果关系; (2) 水体氮浓度 的明显下降, 引起了浮游植物群落结构的变化, 刺激了硅藻、丝状蓝藻占比增加, 促进了水体浮游植物叶绿 素 $a$ 浓度的升高. 如果是这种机制, 氮降低带来的叶绿素 $a$ 浓度升高, 虽然具有因果关系, 但主要是非水华 种藻类的增加. 这种可能性可以从近年来太湖直链硅藻等硅藻门生物量的快速增加得到支持 ${ }^{[30]}$. 初步判 断, 可能是两种机制共同导致了太湖目前 DTN 浓度与水体叶绿素 $a$ 浓度的负相关关系. 比较几个营养盐参 数发现, 15 年来太湖冬春季 $\mathrm{DTN}_{12-3}$ 浓度与 $[\mathrm{Chl} . a]_{\mathrm{LB}}$ 之间统计关系最好, 因此, 可以在蓝藻水华强度的预测 中将 $\mathrm{DTN}_{12-3}$ 列入作为营养盐要素代表.

使用温度和降水指标作为太湖年度蓝藻水华情势预测的关键参数既符合太湖实际, 也具有合理性. 近 年来,变暖、极端天气增多等气候变化与人类活动的影响交织,使得全球湖泊、水库等淡水水体蓝藻水华问 题在加重. Huisman 等系统分析了近年来气候变化趋势有利于蓝藻水华发生的机制,包括: (1)气温升高、热 分层加剧, 更有利于喜欢表层生长的蓝藻生长和细胞集聚, 且下层缺氧几率增大带来的内源营养盐释放增 加; (2) 大气 $\mathrm{CO}_{2}$ 分压增高, 增加水体 $\mathrm{CO}_{2}$ 供给, 有利于水体浮游植物生长; (3) 暴雨等极端气象事件增加带 来的流域地表冲刷作用加剧, 湖泊外源营养盐负荷增加等 ${ }^{[32]}$. Huisman 提出的气候变化有利于蓝藻水华的 几种机制, 近年来在太湖都有所表现. 施坤等通过太湖 2003-2017 年蓝藻水华物候学分析发现, 气候变暖 等导致近年来太湖蓝澡水华发生的物候学条件提前了近 1 个月 ${ }^{[26]}$. 邓建明等研究发现, 气温升高显著影响 了太湖浮游植物群落结构的演替, 气温、水温与微囊藻生物量的变化呈显著正相关 ${ }^{[33]}$. 近年来全球普遍发 生的风速下降现象在太湖流域同样存在, 而风速的下降有利于易于上浮集聚的蓝藻细胞快速增殖和优势形 

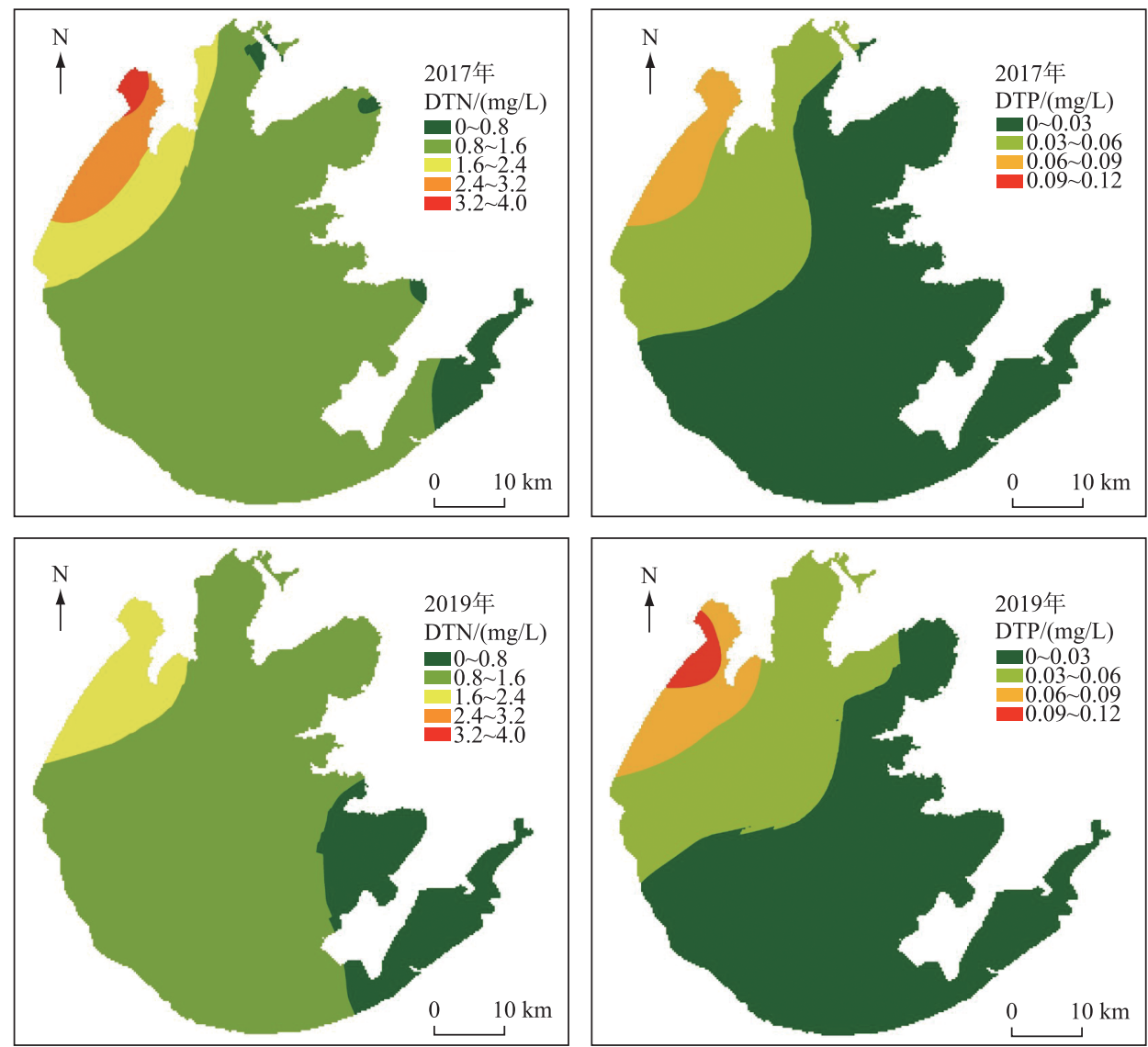

图 52017 年与 2019 年太湖年均 DTN 和 DTP 空间插值对比

Fig.5 Comparison of spatial DTN and DTP distribution between 2017 and 2019 in Lake Taihu

成, 叠加太湖近年来的水位升高, 下层水体扰动减弱, 又促进了太湖内源释放, 共同加剧了太湖的蓝藻水华 问题 ${ }^{[34]}$.

冬、春季水温的变化对微囊藻为主的蓝藻冬季种源、春季生物量基础有着重要的影响. 因此, 水温成为 预测年度蓝藻水华情势的重要考量要素是合理的. 平均温度显示出比有效积温更好的蓝藻水华强度拟合关 系, 说明太湖的蓝藻生长、蓝藻水华强度不仅受 $9^{\circ} \mathrm{C}$ 以上的水温高低的影响, 更低的温度对藻类种源的存量 基础也有影响. 其原因可能有两点: (1) 太湖的微囊藻在 $9^{\circ} \mathrm{C}$ 以下死亡或生物量下降是个缓慢的过程, 甚至 成团之后的微囊藻能在 $9^{\circ} \mathrm{C}$ 以下长期存在. 马健荣等发现太湖冬季蓝藻水华还能存在, 表明短期低温下微囊 藻依然存活、微囊藻水华尚可存在 ${ }^{[35]}$. 此外, 如果低于 $9^{\circ} \mathrm{C}$ 时微囊藻细胞能够部分被冻死, 抑或有助于食物 链牧食降低微囊藻种源, 那么, 温度低于 $9^{\circ} \mathrm{C}$ 也的确依然影响整个冬季的蓝藻生物量基础. (2) 其他藻类的 生长状况会间接影响夏季微囊藻的生长. 比如, 冬季太湖的鱼腥藻大量存在, 其生物量对后期微囊藻的演替 具有影响. 此外, 冬季硅藻生物量偏高, 可能有助于营养盐在水相的累积, 也有利于春、夏季微囊藻生长. 因 此,选择 $\mathrm{WT}_{12-3}$ 而非有效积温作为温度预测变量, 对太湖蓝藻水华年度强度的预测更为合理.

降雨量高低对太湖蓝藻水华形成和发展的影响是多方位的. 太湖的降雨量不但代表了太湖的年度换水 程度, 还在很大程度上反映了外源综合污染的负荷量. 翟淑华等通过水量平衡及物量平衡的方法, 估算了太 湖 2012 年、2015-2016 年的磷负荷特征, 指出人湖水量大小是影响太湖磷收支的主要因素 ${ }^{[36-37]}$. 朱伟等进 一步分析认为 2016 年太湖流域特大洪水期间的洪峰携带人湖及太湖的高滞留特点对太湖磷收支影响很 
$大^{[38]}$. 陈洁等分析了 2017 年 3 月 1 日一 2018 年 2 月 28 日间太湖主要营养盐人湖河道大浦河 (陈东港) 水体 氮、磷浓度与降雨过程之间的关系 ${ }^{[39]}$, 发现河道人湖水量是表征磷负荷的重要指标, 河道降雨过程对人湖河 道水体氮、磷浓度影响不大,大雨、中雨、小雨和无雨期间河道水体总氮浓度分别为 $3.00 、 3.34 、 3.55$ 和 3.37 $\mathrm{mg} / \mathrm{L}$, 总磷浓度分别为 $0.228 、 0.258 、 0.219$ 和 $0.225 \mathrm{mg} / \mathrm{L}$, 不同雨强之间统计上无差异, 降雨量、人湖水量很 大程度上表征了河道人湖的氮、磷负荷. 此外, 降雨量大小还影响了太湖湖体的营养盐等物质的空间输移, 间接影响蓝藻适宜生长区的时空动态变化. 比如 2016 年的暴雨增加了太湖高磷浓度水域的空间扩张 ${ }^{[38]}$, 形 成了明显的自西北向东南湖区推移趋势, 增加了蓝藻水华易发区的面积, 从而影响蓝藻水华情势. 因此, 降 雨量大小综合反映了水文和营养盐两方面对蓝藻水华情势的影响,纳人预测模型具有合理性. 由于秋、冬季 降雨量不大, 偶然性很大, 因此利用前一年总降雨量 $\mathrm{RF}_{\mathrm{YB}}$ 对年度蓝藻水华强度的预测精度反而明显高于秋、 冬季 $\mathrm{RF}_{9-2}$ 或冬、春季 $\mathrm{RF}_{12-3}$.

\section{2 预测模型存在的不足}

缺乏对未来气象情景预测信息的综合考虑是本预测模型的不足. 本预测模型主要从影响藻类生长的现 有环境条件基础的角度开展未来藻情预测. 影响藻情变化的, 除了现有条件基础, 还与未来春、夏季蓝藻水 华暴发期间的水文、气象、营养盐条件密切关联. 因此,对未来春、夏季气象水文情势的预测信息未能体现， 是本预测模型的不足. 目前对气候预测的信息渠道比较多,有一些非常专业的机构对外发布报告,可将其纳 人,将有助于对未来蓝藻水华情势的判断.

缺乏对藻类群落结构变化的深人分析会影响本预测模型的精度. 本预测也未考虑藻类群落结构信息的 影响, 泛泛采用在一定程度上反映总藻类生物量的叶绿素 $a$ 浓度年均值表征蓝藻水华强度,在藻情评估方 法上尚有一定的不足. 太湖藻类群落结构的季节变化较大,空间差异性也很大 ${ }^{[30]}$,因此,仅仅采用藻类叶绿 素 $a$ 浓度表征有害蓝藻生物量、微囊藻生物量存在一定的不足. 目前, 由于藻类群落结构数据的监测、可比 性仍待提高,优质数据的获取存在难度,纳人模型之后可能会降低预测工作的时效性. 建议在今后模型的优 化中,当藻类群落结构快速检测技术成熟时 ${ }^{[40]}$, 将其纳人预测模型中.

致谢: 太湖湖泊生态系统研究站钱荣树、黄建明、韦金权、夏丽萍等及时提供了 2019 年监测数据,在此表示 感谢.

\section{4 参考文献}

[ 1 ] Qin B, Paerl HW, Brookes JD et al. Why Lake Taihu continues to be plagued with cyanobacterial blooms through 10 years (2007-2017) efforts. Science Bulletin, 2019, 64(6) : 354-356.

[ 2 ] Zhang M, Shi X, Yang Z et al. The variation of water quality from 2012 to 2018 in Lake Chaohu and the mitigating strategy on cyanobacterial blooms. J Lake Sci, 2020, 32(1): 11-20. DOI: 10.18307/2020.0102. [张民, 史小丽, 阳振等. 2012-2018 年巢湖水质变化趋势分析和蓝藻防控建议. 湖泊科学, 2020, 32(1) : 11-20.]

[ 3 ] Guo C, Zhu G, Paerl HW et al. Extreme weather event may induce Microcystis blooms in the Qiantang River, Southeast China. Environmental Science and Pollution Research, 2018, 25: 22273-22284

[ 4 ] Michalak AM, Anderson EJ, Beletsky D et al. Record-setting algal bloom in Lake Erie caused by agricultural and meteorological trends consistent with expected future conditions. PNAS, 2013, 110(16) : 6448-6452.

[ 5 ] Steffen MM, Davis TW, McKay RML et al. Ecophysiological Examination of the Lake Erie Microcystis bloom in 2014: Linkages between biology and the water supply shutdown of Toledo, OH. Environmental Science \& Technology, 2017, 51: 6745-6755.

[ 6 ] Namsaraev Z, Melnikova A, Ivanov V et al. Cyanobacterial bloom in the world largest freshwater Lake Baikal. IOP Conf. Series: Earth and Environmental Science, 2018, 121: 032039.

[ 7 ] Ho JC, Michalak AM, Nima Pahlevan N. Widespread global increase in intense lake phytoplankton blooms since the 1980s. Nature, 2019, 574: 667-670.

[ 8 ] Schofield O, Grzymski J, Bissett WP et al. Optical monitoring and forecasting systems for harmful algal blooms: Possibiligy or pipe dream? Journal of Phycology, 1999, 35: 1477-1496.

[ 9 ] Wong KTM, Lee JHW, Hodgkiss IJ. A simple model for forecast of coastal algal blooms. Estuarine, Coastal and Shelf Science, 2007, 74: 175-196. 
[10] Freeman KS. Forecasts aid HABs response. Environmental Health Perspectives, 2011, 119(12) : A510.

[11] Tian Y, Huang M. An integrated web-based system for the monitoring and forecasting of coastal harmful algae blooms: Application to Shenzhen City, China. Journal of Marine Science and Engineering, 2019, 314 ( 7): DOI: 10. 3390/ jmse7090314.

[12] Kong FX, Ma RH, Gao JF et al. The theory and practice of prevention, forecast and warning on cyanobacterial bloom in Lake Taihu. J Lake Sci, 2009, 21(3) : 314-328. DOI: 10.18307/2009.0302. [孔繁翔, 马荣华, 高俊峰等. 太湖蓝藻 水华的预防预测和预警的理论与实践. 湖泊科学, 2009, 21(3): 314-328.]

[13] Qin BQ, Zhu GW, Zhang YL et al. The Application of a high-frequency, wireless and automatic observation technique in algal bloom monitoring and forecasting in eutrophic lake. E-Science: Technology and Application, 2010, 1(3) : 61-70. [秦 伯强, 朱广伟, 张运林等. 高频无线自动监测技术在湖泊蓝藻水华监测和预测中的应用. 科研信息化技术与应用, $2010, \mathbf{1}(3)$ : 61-70.]

[14] Li W, Qin B, Zhu G. Forecasting short-term cyanobacterial blooms in Lake Taihu, China, using a coupled hydrodynamicalgal biomass model. Ecohydrology, 2014, 7: 794-802.

[15] Qin B, Li W, Zhu G et al. Cyanobacterial bloom management through integrated monitoring and forecasting in large shallow eutrophic Lake Taihu (China). Journal of Hazardous Materials, 2015, 287: 356-363.

[16] Ye L, Cai Q. Forecasting daily chlorophylla concentration during the spring phytoplankton bloom period in Xiangxi Bay of the Three-Gorges Reservoir by means of a recurrent artificial neural network. Journal of Freshwater Ecology, 2009, 24 (4) : 609-617.

[17] Zhang H, Hu W, Gu K et al. An improved ecological model and software for short-term algal bloom forecasting. Environmental Modelling \& Software, 2013, 48: 152-162.

[18] Xiang B, Zhao Y, Wang X et al. Research on algae blooms forecasting based on the multivariate data driven method: a case study of the Chaohu Lake. IOP Conf. Series: Earth and Environmental Science, 2016, 46: 012044.

[19] Xiao X, He J, Huang H et al. A novel single-parameter approach for forecasting algal blooms. Water Research, 2017, 108: 222-231.

[20] Wang CY, Yu Y, Sun YK et al. The discussion of the early forecasting of cyanobacteria bloom in the Lake Taihu based on ELCOM-CAEDYM model. China Environmental Science, 2013, 33(3)：491-502. [王长友, 于洋, 孙运坤等. 基于 ELCOM-CAEDYM 模型的太湖蓝藻水华早期预测探讨. 中国环境科学, 2013, 33(3) : 491-502.]

[21] Deng JM, Qin BQ, Wang BW. Quick implementing of generalized additive models using R and its application in bluegreen algal bloom forecasting. Chinese Journal of Ecology, 2015, 34(3): 835-842. [邓建明, 秦伯强, 王博雯. 广义可加模型 在 R 中的快捷实现及蓝藻水华预测分析中的应用. 生态学杂志, 2015, 34(3)：835-842.]

[22] Stumpf RP, Wynne TT, Baker DB et al. Interannual variability of cyanobacterial blooms in Lake Erie. PLoS ONE, 2012, $7(8)$ : e42444.

[23] Stumpf RP, Johnson LT, Waynne TT et al. Forecasting annual cyanobacterial bloom biomass to inform management decisions in Lake Erie. Journal of Great Lake Research, 2016, 42: 1176-1183.

[24] Obenour DR, Gronewold AD, Stow CA et al. Using a Bayesian hierarchical model to improve Lake Erie cyanobacteria bloom forecasts. Water Resource Research, 2014, 50: 7847-7860.

[25] Zhu GW, Zou W, Guo CX et al. Long-term variations of phosphorus concentration and capacity in Lake Taihu 20052018: Implications for future phosphorus reduction target management. J Lake Sci, 2020, 32 (1) : 21-35. DOI: 10.18307/ 2020.0103. [ 朱广伟, 邹伟, 国超旋等. 太湖水体磷浓度与赋存量长期变化 (2005-2018) 及其对未来磷控制目标 管理的启示. 湖泊科学, $2020,32(1): 21-35$.]

[26] Shi K, Zhang Y, Zhang Y et al. Phenology of phytoplankton blooms in a trophic lake observed from long-term MODIS data. Environmental Science \& Technology, 2019, 53: 2324-2331.

[27] Shi K, Zhang Y, Zhou Y et al. Long-term MODIS observations of cyanobacterial dynamics in Lake Taihu: Responses to nutrient enrichment and meteorological factors. Scientific Reports, 2017, 7: 40326.

[28] Xu H, Paerl H, Qin B et al. Determining critical nutrient thresholds needed to control harmful cyanobacterial blooms in eutrophic Lake Taihu, China. Environmental Science \& Technology, 2015, 49: 1051-1059.

[29] Zhu GW, Qin BQ, Zhang YL et al. Variation and driving factors of nutrients and chlorophyll-a concentrations in northern region of Lake Taihu, China, 2005-2017. J Lake Sci, 2018, 30(2) : 279-295. DOI: 10.18307/2018.0201. [朱广伟, 
秦伯强, 张运林等. 2005-2017 年北部太湖水体叶绿素 a 和营养盐变化及影响因素. 湖泊科学, 2018, 30(2) : 279-295. ]

[30] Guo C, Zhu G, Qin B et al. Climate exerts a greater modulating effect on the phytoplankton community after 2007 in eutrophic Lake Taihu, China: Evidence from 25 years of recordings. Ecological Indicators, 2019, 105: 8-91.

[31] Wang JH, He LQS, Yang C et al. Comparison of algal bloom related meteorological and water quality factors and algal conditions among Lake Taihu, Chaohu, and Dianchi (1981-2015). J Lake Sci, 2018, 30(4) : 897-906. DOI: 10.18307/ 2018.0403. [王菁晗, 何吕奇姝, 杨成等. 太湖、巢湖、滇池等水华与相关气象、水质因子及其响应的比较 (19812015 年). 湖泊科学, $2018, \mathbf{3 0}(4): 897-906$.

[32] Huisman J, Codd GA, Paerl HW et al. Cyanobacterial blooms. Nature Reviews: Microbiology, 2018, 16: 471-483.

[33] Deng J, Qin B, Paerl HW et al. Effects of nutrients, temperature and their interactions on spring phytoplankton community succession in Lake Taihu, China. PLoS ONE, 2014, 9(12) : e113960.

[34] Deng J, Paerl HW, Qin B et al. Climatically-modulated decline in wind speed may strongly affect eutrophication in shallow lakes. Science of the Total Environment, 2018, 645: 1361-1370.

[35] Ma J, Qin B, Paerl HW et al. The persistence of cyanobacterial (Microcystis spp.) blooms throughout winter in Lake Taihu, China. Limnology and Oceanography, 2016, 61: 711-722.

[36] Zhai SH, Han T, Chen F. Self-purification capacity of nitrogen and phosphorus of Lake Taihu on the basis of mass balance. J Lake Sci , 2014, 26(2) : 185-190. DOI : 10.18307/2014.0203. [翟淑华, 韩涛, 陈方. 基于质量平衡的太湖氮、磷自 净能力计算. 湖泊科学, $2014,26(2): 185-190$. ]

[37] Zhai SH, Zhou Y, Cheng YH et al. Calculation of total phosphorus loads from rivers around Lake Taihu and analysis of total phosphorus fluctuation in the lake in 2015-2016. J Lake Sci, 2020, 32(1) : 48-57. DOI: 10.18307/2020.0105. [翟 淑华, 周娅, 程媛华等. 2015-2016 年环太湖河道进出湖总磷负荷量计算及太湖总磷波动分析. 湖泊科学, 2020, 32( 1$)$ : 48-57.]

[38] Zhu W, Hu SY, Feng GY et al. Effects of great floods on phosphorus in shallow lakes: A case study of Lake Taihu in 2016. J Lake Sci, 2020, 32(2) : 325-336. DOI: 10.18307/2020.0201. [朱伟, 胡思远, 冯甘雨等. 特大洪水对浅水 湖泊磷的影响: 以 2016 年太湖为例. 湖泊科学, 2020, 32(2) : 325-336.]

[39] Chen J, Zhu GW, Xu H et al. Influence of rainfall intensity on the nutrient loading from an inflowing river in plain river network of Taihu catchment. Environmental Science, 2019, 40(11): 4924-4931. [陈洁, 朱广伟, 许海等. 不同雨强对 太湖河网区河道入湖营养盐负荷影响. 环境科学, 2019, 40(11) : 4924-4931. ]

[40] Yang J, Holbach A, Wilhelms A et al. Highly time-resolved analysis of seasonal water dynamics and algal kinetics based on in-situ multi-sensor-system monitoring data in Lake Taihu, China. Science of the Total Environment, 2019, 660: 329-339. 